\title{
Soigner autrui, une sinécure ?
}

\section{Is working as a health care professional a sinecure?}

\author{
M.-F. Bacqué \\ (C) Springer-Verlag France 2011
}

La France a mal au travail. Depuis une dizaine d'années, cette expression a fait florès. Les douloureux suicides sur le lieu même des entreprises se sont multipliés et les plaintes ont enfin été relayées par les médecins du travail et les syndicats. Cependant, les conditions de travail ont changé dans un contexte beaucoup plus large, celui de la mondialisation et de la globalisation. Que s'est-il passé pour les soignants ?

Lise Fillion, professeure en sciences infirmières à l'université de Laval au Québec, nous permet d'avoir une vue transatlantique du problème. Des équipes françaises nous proposent de réfléchir également aux améliorations à proposer du côté des jeunes oncologues de Caen et des services d'oncohématologie avec le Groupe de réflexion sur l'accompagnement et les soins palliatifs en hématologie (GRASPH), l'un des fondateurs de l'Association francophone des soins oncologiques de support (AFSOS). Des études développées par ces médecins et psychologues du travail portent aussi bien sur l'amélioration de la qualité de vie au travail des soignants [1,2] que sur le classique burnout de Christina Maslach. Enfin, les idéations suicidaires et le suicide des médecins sont largement abordés comme une conséquence spectaculaire de la pression au travail, doublée de la diminution de l'estime de soi chez les praticiens, qu'ils soient généralistes, réanimateurs ou chirurgiens hospitaliers. Les médecins restent malgré tout considérés comme ayant peu de besoins sur le plan émotionnel. Ainsi, Jean-Paul Hamon, président de l'union généraliste cité par Delfieu [3], notait : « en 18 mois, de 2008 à 2009, il y a eu 23 suicides chez France Télécom pour 190000 salariés. Le directeur de France Télécom est à juste titre sommé par le gouvernement de modifier sa gestion du personnel. En parallèle, chez les médecins, on constate qu'il y a eu plus de 60 suicides parmi les 200000 confrères en exercice ». La prise de conscience de l'épuisement professionnel des soignants et des médecins passe donc bien par le prisme des représentations sociales du travail chez nos concitoyens.

\section{M.-F. Bacqué ( $\square)$}

Rédactrice en chef de Psycho-Oncologie,

$\mathrm{PhD}$, Pr, département de psychologie, université de Strasbourg,

12, rue Goethe, F-67000 Strasbourg, France

e-mail : mfbacque@club-internet.fr

\section{Pourquoi les interventions psychologiques isolées ne marchaient-elles pas ?}

Dès les années 1980, des psychanalystes et des psychologues de formation analytique proposaient un travail clinique auprès des patients hospitalisés. Cette approche diminuait considérablement les conséquences de l'implication émotionnelle des soignants tout en leur permettant de conserver des relations de qualité avec les patients. Cependant, ces interventions entraînaient une forme de séparation entre, d'un côté, des soins somatiques prodigués par médecins et soignants et, de l'autre, des soins psychiques apportés par des spécialistes. Une approche plus globale était dès lors envisagée empiriquement à partir des travaux sur les groupes (M. Balint, D. Anzieu), en introduisant les groupes de parole de soignants, mais aussi les analyses de pratiques régulières. L'observation à distance de toutes ces tentatives pour améliorer l'accompagnement des patients ne se traduisait pourtant pas par une meilleure vie au travail de tous les soignants qui en bénéficiaient.

\section{Épuisement au travail : croisement de facteurs personnels, professionnels et politiques}

L'approche psychologique de la relation soignant-malade est en effet insuffisante. Et cela n'est devenu une certitude que depuis une dizaine d'années. La plupart des recherches se sont d'abord centrées sur les facteurs personnels d'épuisement. Les personnalités immatures, marquées par une idéalisation du métier, l'incapacité à garder la «bonne distance » et par des crises personnelles récentes (deuils, séparations) sont beaucoup plus à risque que les personnes d'expérience, bien insérées sur un plan familial et social. La question du sens du travail, ici traitée par l'équipe de Mélanie Vachon, est une des composantes existentielles dépendante de l'histoire de vie des soignants, mais aussi bien sûr de leur maturité développementale.

Les facteurs professionnels sont liés à la formation des soignants et à la nature du travail dans l'unité (côtoyer, voir, toucher de jeunes patients leucémiques n'a pas les mêmes conséquences qu'avec des grands brûlés) et enfin aux rapports entre les soignants dans l'équipe. C'est surtout à ce niveau que les psychologues, particulièrement en oncologie, travaillent. 
Les groupes de parole permettent de relativiser l'impression de répétition pénible produite par l'hospitalisation d'un nouveau patient pour lequel on peut, d'ores et déjà, pronostiquer une fin de vie difficile. Le fait d'échanger sur le vécu d'une nouvelle relation permet également en groupe de retrouver chez autrui ses propres défenses et d'exposer les représentations inconscientes qui président à certains préjugés. Enfin, les conflits interprofessionnels peuvent trouver des compromis afin de faciliter la poursuite des relations ou le départ vers d'autres fonctions [4].

\section{La valorisation du métier ne compense plus la charge de travail}

Philippe Colombat et son équipe soulignent, pour les services d'oncohématologie, combien la charge de travail n'est plus compensée aujourd'hui par la reconnaissance professionnelle. Ce facteur actuel relève d'un mouvement global de perte de repère par rapport aux professions soignantes. Le paternalisme a cédé le pas devant l'information et une tentative de responsabilisation du patient. La réalité montre cependant que le temps consacré aux questions des patients est moindre, obéré par une charge de travail croissante chez tous les soignants.

Enfin, les facteurs organisationnels touchent toutes les professions soignantes : chez les infirmiers et aides-soignants, il semble que l'hésitation engendrée par l'absence de protocole lors d'un soin difficile ait d'importantes répercussions sur le stress au travail. Chez les médecins, cette dimension est plus ambiguë, car si les protocoles ont une dimension facilitatrice, nombreuses sont les situations singulières de patients pour lesquelles une solution unique doit être trouvée. Pour tous, la dimension aléatoire des soins est épuisante. La planification semble une issue « sur le papier », mais elle est parfois génératrice de rigidité et peut être tournée en dérision lorsqu'elle $s$ 'avère trop souvent inutile.

\section{Plus de transparence dans la direction des équipes soignantes}

Si la confrontation avec des personnes souffrantes, qui plus est, mourantes, est aujourd'hui reconnue comme facteur d'épuisement au travail chez les soignants, il est bien évident que la translation de cet aspect dans d'autres professions non sanitaires ne peut être effectuée. Et pourtant, ces autres professions pâtissent, elles aussi d'un système écrasant. Bien au-delà de la charge de travail, le traitement politique du travail, avec ses aspects hiérarchiques, motivationnels et de partage de l'activité doit dorénavant être abordé.

Le management participatif semble faire ses preuves en oncohématologie en démultipliant les niveaux de rencontres et d'échanges. Les staffs pluriprofessionnels abordent les questions médicales posées par les entrants. L'analyse des pratiques peut avoir lieu autour de groupes Balint ou dans des réunions de morbimortalité où les dysfonctionnements de la prise en charge médicale et soignante ont été marqués. Enfin, les aspects relationnels et le soutien des équipes peuvent être discutés lors de réunions de crise spécifiques ou plus régulièrement dans les groupes de parole (mais ceux-ci reposent sur l'engagement à y participer régulièrement). Notons ici que, si les groupes de parole animés par des psychologues avaient, dans les années 1985, été considérés comme « la » solution dans les services d'oncologie, ils ne pouvaient vraiment pas constituer le levier unique de l'amélioration. Certains psychologues ou analystes de l'époque ont pu cependant se trouver en échec, alors que leur investissement était authentique. Il était tout simplement présomptueux et insuffisamment documenté de croire que la seule libération de la parole suffirait à combler les différents niveaux de frustration.

La direction d'une équipe de soins aigus ou palliatifs qui permet un véritable maillage des personnels et des fonctions doit accepter que longitudinalement et transversalement, l'analyse du travail ait lieu. Un tel partage ne remet pas pour autant en cause la nécessité de prendre des décisions par un chef de service. Encore moins le fait de les assumer. La participation de tous est une manière de partager les responsabilités et de redonner de l'autonomie aux différentes professions. Au travers d'un projet de service, cette démarche peut prendre corps.

Mais pour ces propositions qui pourtant figurent dans le manuel d'accréditation des établissements de la Haute Autorité de santé (HAS), combien vont déboucher sur un apprentissage du management d'équipe soignante ? Les médecins recevront-ils au moins le minimum de formation à la relation? L'habitude de pratiquer le questionnement sur ses propres représentations est-elle donnée à nos soignants ? Ceux qui deviennent cadres ont-ils réellement les moyens d'orienter leurs équipes vers l'autonomie de leur fonction et de leurs tâches? Les évaluations de l'intérêt de ces nouvelles politiques porteront-elles un jour sur le bien-être des soignants mais aussi sur celui des patients?

Psycho-Oncologie vous apporte aujourd'hui les plus récents résultats dans le domaine de la souffrance au travail des soignants et des médecins, à vous de mettre en place ces propositions complexes mais orientées vers la diminution de la souffrance au travail et l'amélioration des conditions du soin hospitalier.

\section{Références}

1. Pronost AM, Le Gouge A, Leboul D, et al (2008) Effet des caractéristiques des services d'oncohématologie développant la démarche palliative et des caractéristiques sociodémographiques des soignants sur les indicateurs de santé : soutien social, stratégies de coping et qualité de vie au travail. Oncologie 10:125-34

2. Blanchard P, Truchot D, Albiges-Sauvin L, et al (2010). Prevalence and causes of burnout amongst oncology residents: a comprehensive nationwide cross-sectional study. Eur J Cancer 15:2708-15

3. Delfieu D (2009) Exercer la médecine, un plaisir ? Actes du $38^{\mathrm{e}}$ Congrès de la Société médicale Balint. Strasbourg, 55-9

4. Delbrouck M (2008) (dir) Le syndrome d'épuisement professionnel. De Boeck, Bruxelles 